\title{
Microstrip coupling techniques applied to thin-film Josephson junctions at microwave frequencies
}

Sørensen, O H; Pedersen, Niels Falsig; Mygind, Jesper; Dueholm, Benny; Finnegan, T; Hansen, J; Lindelof, $\mathrm{P}$.

Published in:

I E E E Transactions on Magnetics

Link to article, DOI:

10.1109/TMAG.1981.1060965

Publication date:

1981

Document Version

Publisher's PDF, also known as Version of record

Link back to DTU Orbit

Citation (APA):

Sørensen, O. H., Pedersen, N. F., Mygind, J., Dueholm, B., Finnegan, T., Hansen, J., \& Lindelof, P. (1981). Microstrip coupling techniques applied to thin-film Josephson junctions at microwave frequencies. I $E E E$ Transactions on Magnetics, 17(1), 107-110. https://doi.org/10.1109/TMAG.1981.1060965

\section{General rights}

Copyright and moral rights for the publications made accessible in the public portal are retained by the authors and/or other copyright owners and it is a condition of accessing publications that users recognise and abide by the legal requirements associated with these rights.

- Users may download and print one copy of any publication from the public portal for the purpose of private study or research.

- You may not further distribute the material or use it for any profit-making activity or commercial gain

- You may freely distribute the URL identifying the publication in the public portal 
MICROSTRIP COUPLING TECHNIQUES APPLIED TO THIN-FILM JOSEPHSON JUNCTIONS AT MICROWAVE FREQUENCIES

O.H. Soerensen, N.F. Pedersen, J. Mygind, B. Dueholm The Technical University of Denmark, DK-2800 Lyngby

T.F. Finnegan*, J. Bindslev Hansen, P.E. Lindelof

H.C. Ørsted Institute, University of Copenhagen, DK-2100

\section{Summary}

Three different schemes for coupling to low impedance Josephson devices have been investigated. They all employ superconducting thinfilm microstrip circuit techniques. The schemes are: (i) a quarterwave stepped impedance transformer, (ii) a microstrip resonator, (iii) an adjustable impedance transformer in inverted microstrip. Using single microbridges to probe the performance we found that the most primising scheme in terms of coupling efficiency and useful bandwidth was the adjustable inverted microstrip transformer.

\section{Introduction}

The succesful application of Josephson junctions in high frequency systems depends upon how efficiently the devices are coupled to external microwave lines. The Josephson elements are often very low impedance devices, e.g., microbridges or high current-density tunnel junctions. This accentuates the demand for coupling schemes of low characteristic impedance at high frequencies. In applications such as the fluxon oscillator ${ }^{1}$ a single, long junction must be matched. Also in other Josephson microwave oscillators with single junctions ${ }^{2}$ or coherent arrays ${ }^{3}$, it is advantageous to operate at low impedance levels as shown by the following estimate. If a N-junction array is matched to an external load, $\mathrm{R}_{\mathrm{I}}=\mathrm{NR}_{\mathrm{J}}$, the maximum available power in the high frequency limit ( $\mathrm{V}_{\mathrm{dc}} \gg \mathrm{R}_{\mathrm{J}} \mathrm{I}_{\mathrm{c}}$ ) becomes $P_{N}=N\left(R_{J} I_{C}\right)^{2} / 8 R_{J}$. Since $R_{J} I_{C}$ is a constant, the available power increases inversely proportional to $R_{J}$ (and proportional to $N$ ). The junctions cannot be infinitely closely packed due to mutual heating, and hence the number of junctions which can be accomodated at equivalent positions is limited, and the resulting rf-impedance, $\mathrm{NR}_{\mathrm{J}}$, may remain low.

Finally, in applications such as the parametric reflection amplifier ${ }^{4}$ optimum performance is achieved if the signal feed line has characteristic impedance less than the normal state resistance of the parametric device. As a consequence, it is natural to look for microwave circuits of low impedance, i.e., at the $0.1 \Omega$ to $1 \Omega$ leveI.

Here, we report on three different methods to approach the problem. They all use superconducting thin-film microwave integrated circuit techniques because: the thin-film circuits are produced in the same processes which are used to fabricate the junctions, and because superconducting thin-film circuits have low loss, even at high frequencies. The

*Present address: Physikalisch-Technische Bundesanstalt, Institut Berlin, Abbestrasse $2-12,1000$ Berlin 10, BRD. three schemes are: (i) a quarterwave high/low impedance transformer, (ii) a strip-resonator with the Josephson device mounted at the end or in the center, (iii) a novel approach introducing adjustable impedance in microstrip design. The scheme employs an inverted microstrip transformer coupling. In all three cases single thin-film microbridges of normal state resistances in the range $0.02 \Omega$ to $0.15 \Omega$ were used to probe the performance of the circuits.

\section{Quarterwave high/low transformer}

The thin-film circuit pattern is shown in Fig. 1 (a). The location of the microbridge is indicated by the" $x "$. The circuit was made on a $25.4 \times 25.4 \times 1 \mathrm{~mm}$ soda-glass substrate. The impedance transformer consists of two 2-section $\lambda / 4$ transformers separated by a $\lambda / 2$ section. The first 2-step transformer is broadband with moderate impedance ratio. The $50 \Omega$ input line is transformed into $19 \Omega$. In order to reach the desired low output impedance, the second 2step transformer operates with a very large impedance discontinuity $(122 \Omega)$ such that the overall impedance ratio becomes $60: 1$. The circuit was designed to have a center frequency of $10 \mathrm{GHz}$. The performance of the circuit is illustrated in Fig. 2(a) to (e). In (a) the measured power reflection vs. frequency is shown without microbridge. In (b) a similar recording, made after a $70 \mathrm{~m} \Omega$ bridge was introduced in the circuit, is shown. A comparison shows that the cut and the bridge matches the transformer structure in a $2300 \mathrm{MHz}$ bandwidth at 10.4 GHz. Fig. 2(c) shows the measured input signal necessary to maintain a constant suppression of the supercurrent as the input frequency is varied. Maximum attenuation means maximum power transmitted to the bridge detector. In Fig. $2(d)$ the bridge is biased at a fixed voltage and the receiver frequency is varied. Fach trace in the set of curves in (d) shows the measured Josephson radiation vs. frequency. The frequency dependence of the individual traces is a convolution of the Josephson line-width
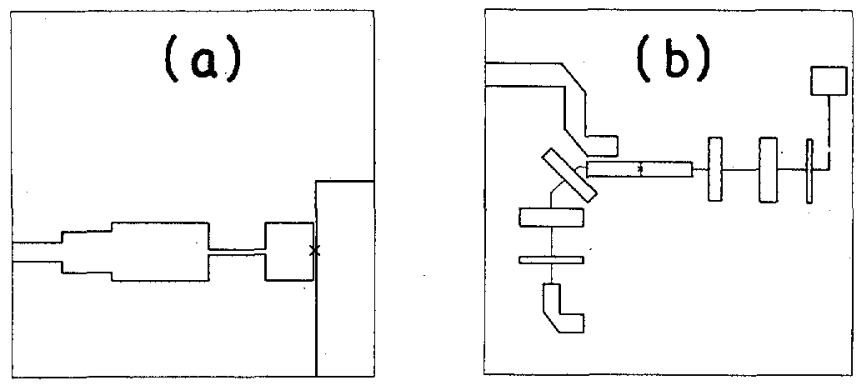

Fig. 1. Thin-film circuit patterns.

(a) 4-section $\lambda / 4$ stepped impedance transformer. (b) Strip resonator with end connected bias leads and side coupled rf-input line. The junction is located at the $x$. 
with the transformer and the receiver response functions. The envelope curve reproduces the transformer/receiver pass-band. The peak positions in (c) and (d) coincides. Finally, (e) shows the calculated power reflection coefficient of the properly terminated transformer structure assuming that all sections are $\lambda / 4$ at $10 \mathrm{GHz}$. The calculated and the measured widths of the reflection minimum are in good agreement. The small disagreement between the calculated and the measured center frequencies are not significant perse since the microstrip design formulae are accurate only to within a few pct. It is not obvious why the $70 \mathrm{~m} s$ bridge behaves as a matched termination when the theoretical matching impedance is $0.9 \Omega$, or why the transmission-peak falls below the reflection-dip. Tentatively, we may understand these results by invoking the well-established facts that microbridges are accompanied by a series inductance ${ }^{2}$ and that the effective lengths of sections of microstrip are influenced by stepin-width discontinuities ${ }^{5}$. In a model calculation we have been able to reproduce the relative frequency shift between reflection and transmission by taking these stray reactances into account.

The maximum integral power detected from a microbridge was $2.4 \mathrm{pw}$ in a $250 \mathrm{MHz}$ linewidth (power density $0.01 \mathrm{pW} / \mathrm{MHz}$ ).

\section{Microstrip resonator}

A different approach to low-impedance coupling is to employ a resonator as impedance transformer. The resonator circuit is shown in Fig. l(b). The high-impedance lines leading to the ends of the resonator section are filtered

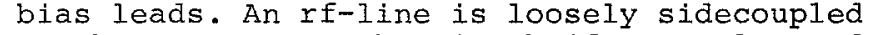
to the resonator. The microbridge was located either in the center (Fig. Ib) or at the end of the resonator. Both conventional and inverted microstrip versions were studied. The resonator length was $\lambda / 2$ at $10 \mathrm{GHz}$ in conventional microstrip. With the bridge at the end, the measured resonance frequencies were $9.5 \mathrm{GHz}$ in conventional and $12.5 \mathrm{GHz}$ in inverted

microstrip respectively. With the bridge in the center, the corresponding resonance frequencies were $13 \mathrm{GHz}$ and $17 \mathrm{GHz}$. In all cases the quality factor was $Q \simeq 200$ as inferred from power reflection measurements. The difference in resonance frequencies between conventional and inverted microstrip simply reflects the different effective dielectric constants. The frequency shift caused by the change in bridge position is not as obvious. It may be understood only if the presence of the bridge (and the $0.5 \mu \mathrm{m}$ wide cut across the resonator) changes the effective length of the resonator. This is consistent with the observation already made that the microbridge is accompanied by a non-negligible series inductance.

Fig. 3 illustrates the performance of the conventional microstrip resonator with a single center-located microbridge. The current-voltage curve (IVC) of the bridge and the emitted Josephson radiation are shown. A cavity induced step is clearly visible in the IVC at 27 $\mu \mathrm{V}$ corresponding to a resonance center frequency of $13.1 \mathrm{GHz}$. With the microwave receiver centered at resonance, a sharp peak in the Josephson radiation is measured as the
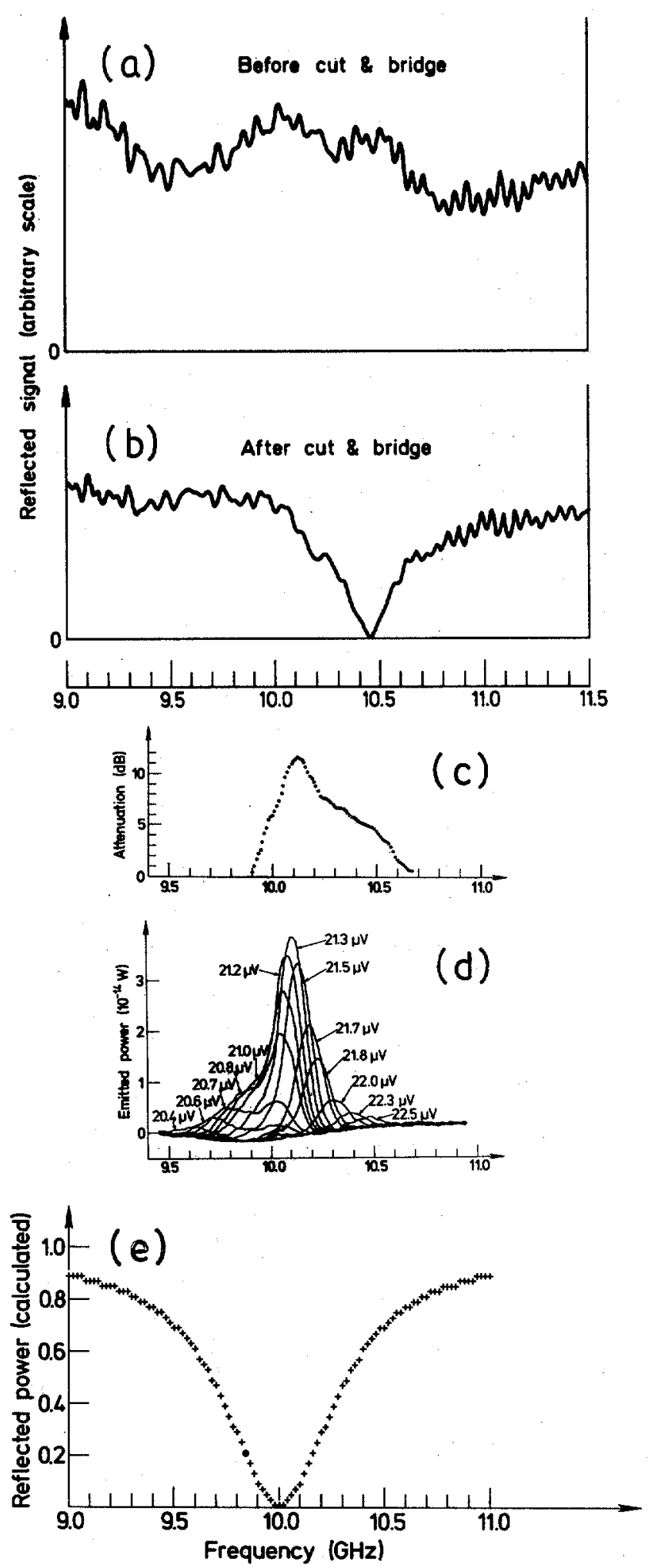

Fig. 2. Performance of multistep transformer. (a) Measured power reflection vs. frequency before scratching bridge. (b) Same as (a) with bridge present. (c) Measured attennuator setting in input line to maintain constant supercurrent suppression. (d) Measured power output from bridge. Each trace corresponds to a fixed dc-bias voltage as indicated. (e) Calculated power reflection coefficient. 


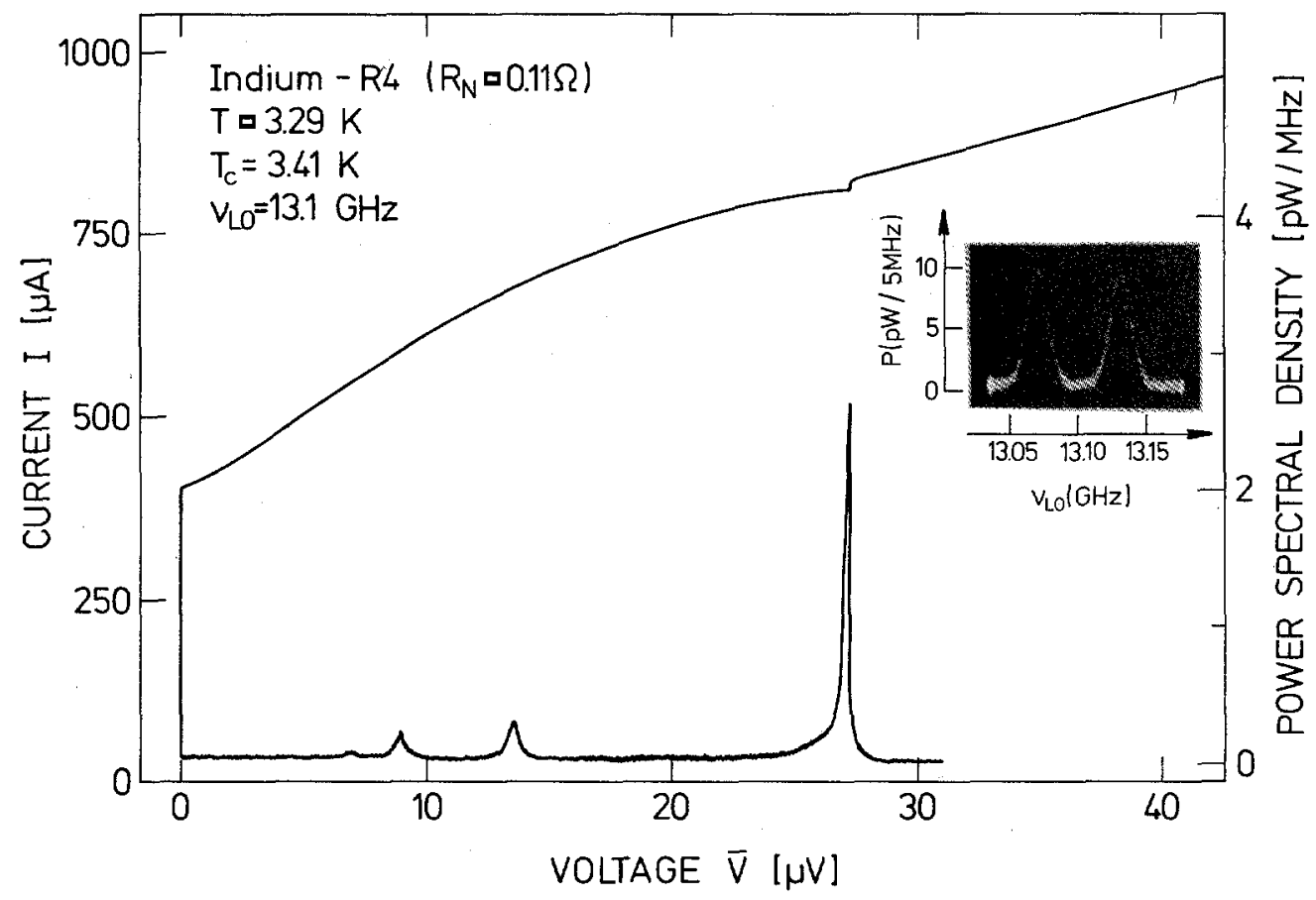

Fig. 3. IV-curve for microbridge in strip resonator and measured spectral density with a fixed receiver frequency (resolution bandwidth $5 \mathrm{MHz}$ ). The inset shows the IF video output vs. LO-frequency. The two receiver sidebands are resolved.

bias voltage is swept past the cavity step. The measured integral power is $50 \mathrm{pW}$ and the linewidth $\sim 20 \mathrm{MHz}$ as seen in the inset, which shows a spectrum analyzer frequency display of the detected Josephson line (the double peak is an artifact of the superheterodyne detection scheme). The maximum integral power measured was $80 \mathrm{pW}(4 \mathrm{pW} / \mathrm{MHz})$. The measured peak in the rf-power vs. voltage curve is skewed due to parametric excitation of the resonator at $13.1 \mathrm{GHz}$ at bias voltages on the low voltage side of the resonator step. Peaks are also seen at voltages where higher harmonics of the Josephson frequency coincide with the receiver and resonator frequency. A similar "skewness" of these harmonic peaks is not observed.

\section{Adjustable inverted microstrip}

\section{transformer}

The third approach to the design of low impedance coupling circuits for use with Josephson junctions employs inverted microstrip. The inverted microstrip has sqeral attractive features, (i) the characteristic impedance is inversely proportional to the strip-to groundplane spacing, (ii) the dielectric medium is vacuum or helium which implies that the stripto-groundplane spacing, and hence the impedance, may be varied and that the dielectric losses are small, (iii) the supporting substrate plays no essential role in the wave propagation and inexpensive substrates may be used. We have designed a microstrip holder with nominal center frequency at 35 GHz. The basic idea is illustrated in Fig. 4. The prototype uses a single $\lambda / 4$ section of inverted microstrip with a substrate-to-groundplane spacing which may be varied by bending the substrate by means of a remotely controlled differential screw and eccentric lever mechanism. With a strip-width of $2 \mathrm{~mm}$ and an air gap of $5 \mu \mathrm{m}$ to $60 \mu \mathrm{m}$ we achieve transformer output impedances in the range $0.1 \Omega$ to $5 \Omega$. The impedance transformation is realized via a $50 \mu \mathrm{m}$ step in the groundplane at a distance $\lambda / 4$ from the Josephson device. The circuit pattern is shown in the upper part of Fig. 4. The bridge is located at the $x$ and is followed by a sequence of $\lambda / 4$ sections which transform the open ended line to a low impedance termination at the bridge. The side arms are dc-bias leads and the input line is dc-ground. The substrate is an inexpensive microscope coverslide $24 \times 24 \times 0.13 \mathrm{~mm}$ which is easily bent. The performance may be described as follows. With a $35 \mathrm{GHz}$ signal applied to the input, the microwave power transmitted to the bridge was measured vs. substrate-togroundplane spacing. The measured fractional power transfer was found proportional to the output impedance of the transformer, calculated as a function of substrate position.6

In Fig. 5 we show the measured incident power (closed symbols) necessary to induce a $10 \%$ suppression of the supercurrent as the frequency is varied with fixed substrate. Also shown is the input power corrected for the measured reflected power (open symbols).

A broadband coupling has been achieved except for a strong resonant absorption peak at 33.5 $\mathrm{GHz}$.

Also the microwave generation by the ac-Josephson effect was measured vs. substrate position and frequency. These measurements were in perfect agreement with the results described above. At optimum as much as $120 \mathrm{pW}$ was extracted from a $40 \mathrm{~m} \Omega$ bridge and about half of that 


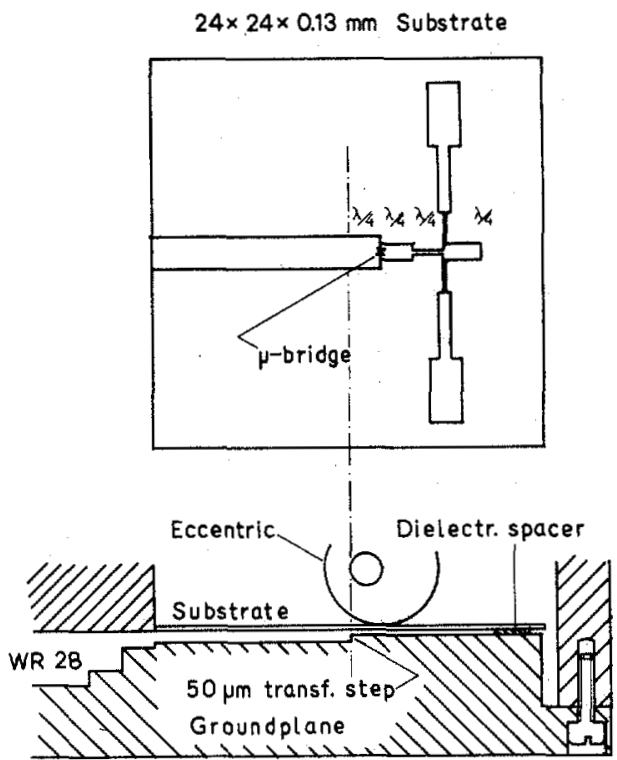

Fig. 4. Upper part: thin-film pattern. Lower part: schematic cross section of the microstrip holder showing the principle of operation.

from a $20 \mathrm{~m} \Omega$ bridge. The linewidth was $10-15$ $\mathrm{MHz}(10 \mathrm{pW} / \mathrm{MHz})$.

\section{Conclusion}

We have found superconducting thin-film microstrip techniques well suited for coupling to low impedance Josephson devices.

The approach using a high/low $\lambda / 4$ impedance transformer was moderately succesful. In order to achieve very low output impedance levels, we have to operate with rather extreme discon-

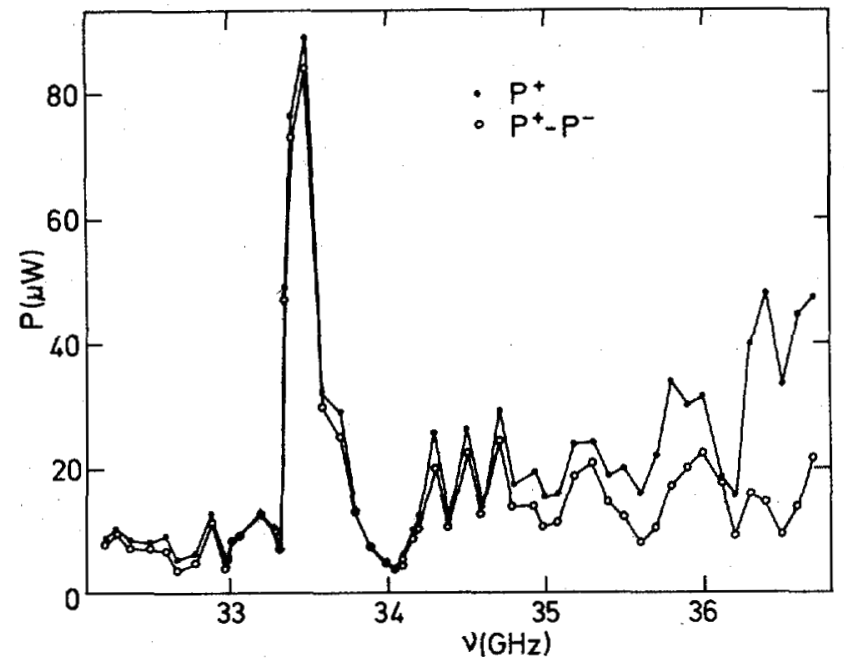

Fig. 5. Measured incident power nenessary to maintain a constant supercurrent suppression (closed circles). Input power corrected for reflection (open circles). tinuities in stripline width. Such large jumps in width require that corrections are made for fringing fields at the edges. (Design equations for microstrip which include these corrections do not apply to the present extreme case.)

The resonator coupling works well in a narrow bandwidth and may be useful in fixed frequency applications. Considerable power was extracted from a single microbridge. Compared to the transformer-coupled case, the emitted linewidth was narrowed by the regenerative interaction between bridge and resonator.

The best performance so far was achieved with the adjustable inverted microstrip transformer coupling. The narrow Josephson line was tunable over a $10 \%$ bandwidth without significant change in output power. The measured power output from a single microbridge was the largest ever reported.

\section{Acknowledgement}

This work was supported in part by the Danish Natural Science Research Council. O.Eg is acknowledged: for the sample preparation.

\section{References}

1. S.N. Erné and R.D. Parmentier, "Microwave radiation from Iong Josephson tunnel junctions". (This conference).

2. N.F. Pedersen et al., "Microwave generation and complex microwave responsivity measurements on small-Dayem bridges". IEEE Trans. on MAG-13, 248 (1977)

3. J. Bindslev Hansen et al., "Coherent arrays of thin film Josephson microbridges". (This conference).

A.K. Jain et al." "Large arrays of Josephson microbridges as tunable sources of microwave radiation". (This conference).

4. M.T. Levinsen et al., "Externally pumped millimeter wave Josephson junction parametric amplifier".

IEEE Trans. on Electr. Devices, Special issue on Josephson junction devices. Oct. 1980

5. K.C. Gupta, Ramesh Garg, I.J. Bahl, Microstrip lines and slotlines, Dedham: Artech House Inc., 1979, p. 136.

6. T. F. Finnegan and O. H. Soerensen, "Adjustable microstrip transformer for use with Josephson devices in millimeter wave integrated circuits", to be published. 